\title{
Impact of EMBODY Experiencing Dementia through New Media Exhibit: Engaging nursing students and the public about dementia through arts-based knowledge translation methods
}

\author{
Kristine Newman ${ }^{1}$, Jacky Au Duong ${ }^{2}$, Parmeet Kahlon*1, Shu Jie Li ${ }^{1}$ \\ ${ }^{1}$ Daphne Cockwell School of Nursing, Ryerson University, Toronto, Canada \\ ${ }^{2}$ Faculty of Communication and Design, Ryerson University, Toronto, Canada
}

Received: September 15, 2019

DOI: $10.5430 /$ jnep.v10n5p100
Accepted: January 16, 2020

Online Published: February 26, 2020

URL: https://doi.org/10.5430/jnep.v10n5p100

\begin{abstract}
Objective: This paper aims to look at the impact of arts-based knowledge translation (ABKT) methods in raising awareness among nursing students and the public about the sex differences and behavioral and psychological symptoms of dementia (BPSD), implemented through a creative new media arts-exhibit at Toronto Rehabilitation Institute.

Methods: Through surveys, interviews, and pre- and post-exhibit questionnaires, this project evaluated the use and efficacy of multi-modal media in translating data from a study on the BPSD. The research team categorized and conceptualized artwork and narratives based on data collected from previous phases; no confidential or identifying information related to study participants were used or displayed in the final exhibit.

Results: The use of photographs, paintings, abstract data visualizations, augmented reality and virtual reality had various levels of effectiveness in engaging nursing students and the public on the topic of dementia. $360^{\circ}$ videos, photographs, and paintings provided the highest level of engagement and discussion among nursing students. The majority of the students reported a better understanding and empathy towards people living with dementia after the viewing of the exhibit and all students perceived the exhibit as an effective method in portraying dementia experience and contributed to their overall understanding of the BPSD.

Conclusions: This knowledge mobilization project overall provided a more informed perspective on BPSD among nursing students and the public, and effectively sparked discussion among viewers. The exhibit was able to raise awareness for dementia, its symptoms, and experiences of patients living with dementia. For the scholarly community, our project presents new ways to mobilize knowledge among a broad audience and demonstrates unique, innovative, and engaging forms of ABKT.
\end{abstract}

Key Words: Dementia, Arts-based knowledge translation, Nursing education, Relational practice, New media

\section{INTRODUCTION}

As the population ages, healthcare provision is becoming increasingly complex, especially with a rise in the prevalence of cognitive issues, like dementia. ${ }^{[1]}$ Dementia is a syndrome with a diverse set of behavioral and psychological symptoms
(BPSD) that can be caused by a variety of neuropsychological disorders, such as Alzheimer's disease or cerebrovascular pathologies. ${ }^{[2]}$ These symptoms vary in their manifestation among different people- with notable differences between sexes — producing a highly subjective experience of cogni-

*Correspondence: Parmeet Kahlon; Email: parmeet.kahlon@ryerson.ca; Address: parmeet.kahlon@ryerson.ca. 
tive BPSD such as agitation. Furthermore, this can cause suffering among people with dementia and stress on their caregivers.

Despite the importance of using evidence to inform practice, there is a consistent evidence-practice gap as research findings are not regularly adopted by healthcare providers. ${ }^{[3]}$ This gap can produce disparities in the care provided to vulnerable populations, such as persons with dementia. This generates a need to look at the different forms of knowledge dissemination to promote increased evidence uptake and application in the field of dementia-related care provision.

The arts-informed approach, or arts-based knowledge translation (ABKT), in health care is an emerging field and involves using rigorous and creative approaches for translating research findings to diverse target audiences. Common arts media employed in arts-based knowledge translation include poetry, images, video, installation art, theatre, dance, and visual arts. ${ }^{[4,5]}$ Literature, mentioned below, has shown that the use of ABKT can help in the understanding and retention of research findings, promoting the uptake of evidence into practice.

This paper evaluates Phase Three of a multiphase study conducted by the authors focused on detecting and predicting the behavioral and psychological symptoms of dementia (BPSD) through innovative tracking technologies. Phases One and Two involved the validation and collection of data regarding BPSD using predictive tracking technology. ${ }^{[6,7]}$ As a case study, our project attempted to facilitate the understanding of complex concepts such as BPSD, the fact that sexes experience BPSD in different ways, and more specifically, to communicate early findings of our project for predicting and detecting agitation of persons with dementia. Using the ABKT approach, the primary outcome of Phase Three was a new media exhibit hosted at Toronto Rehabilitation Institute featuring various creative art forms including photographs, paintings, written word, data visualizations, augmented and virtual reality.

By incorporating various forms of media, we intended to raise awareness of specific conditions by allowing knowledge users to explore and interpret the scenarios and experience various factors. This approach has been widely adopted in the UK (e.g., "A Walk Through Dementia" Alzheimer's Research UK). ${ }^{[8]}$ To our knowledge, however, none of these initiatives have been adapted in the Canadian context, nor have these focused on how a patient's sex influences how the symptoms of agitation are experienced by a person with dementia. Within Canada, ABKT initiatives focused solely on single-form media such as dance, performance, or art. Overall, our study aims to evaluate the effectiveness of multi- modal ABKT in increasing awareness of sex differences and BPSD amongst nursing students and the general public.

\section{Literature review}

Dementia presents with a variety of behavioral and psychological symptoms, including agitation, psychosis, and irritability, that have serious impacts on the quality of life of both patients and caregivers. ${ }^{[9,10]}$ There are prominent differences in the BPSD experienced according to sex; women are more likely to experience depression, anxiety and agitation then men with Alzheimer's disease. ${ }^{[11]}$ Men were found to be more likely to experience aggressiveness and diurnal rhythm issues than women. ${ }^{[12]}$ These findings necessitate the need for awareness and training among healthcare providers and students regarding the appropriate management of sexrelated BPSD.

As of recently, the primary option for treatment of BPSD has been pharmacological, with the prescription of antipsychotics for symptomatic management, despite no significant evidence to support their usage. These, along with other psychotropic medications, have been shown to have significant adverse effects, contributing to increased mortality and risk of cerebrovascular events in patients with dementia. ${ }^{[13]}$ In a study conducted by Cousins, Bereznicki, Cooling and Peterson $(2017),{ }^{[14]}$ it was found that nurses and family members strongly influence medical practitioners towards prescribing psychotropic medications, as nearly half of the sample felt that they required increased training of BPSD to manage symptoms using nonpharmacological methods, and thus reducing the use of psychotropic drugs. Similarly, Jennings et al. $(2018)^{[15]}$ found that general practitioners felt a lack of knowledge of BPSD and their management using multiple treatment modalities, which likely contributes to the heavy reliance on pharmacotherapy.

Compassion for persons with dementia is also variable amongst healthcare students. Compassion and empathy are developed during educational training, which makes it crucial to implement interventions at critical stages to foster their development. ${ }^{[16]}$ A study conducted by Bickford et al. (2019) ${ }^{[16]}$ showed that students primarily reported aggressive BPSD and a lack of knowledge and experience working with patients with dementia as significant barriers to the provision of compassionate care. Zeilig, Poland, Fox and Killick $(2015)^{[17]}$ highlight a serious gap in healthcare provider skills in the provision of dementia care, necessitating the need for additional training. This led to the development of an artsbased workshop by Zeilig et al. (2015) ${ }^{[17]}$ involving the use of music, poetry, film and games, created a safe space for reflective practice and helped staff see persons with dementia positively as individuals with unique experiences. 
Practice is informed by evidence, yet there is a persistent evidence-practice gap that can prevent healthcare providers from changing practice to resolve issues in individualistic and compassionate care provision. ${ }^{[3]}$ In the field of knowledge translation, scientifically rigorous methods of research dissemination have traditionally been merited as superior to other forms, creating a highly linear, restrictive system of sharing research findings that likely contributes to the evidence-practice gap that persists in the healthcare field. ${ }^{[3]}$ Particularly, traditional forms of knowledge dissemination, such as complicated written text and figures, have proven to be inadequate in effectively communicating sensitive knowledge topics that involve a higher degree of subjectivity for comprehension. ${ }^{[18]}$ The evidence-practice gap is particularly evident in research conducted in the social sciences that deal with subjective, experiential topics, such as dementia. This makes it crucial to employ innovative knowledge translation methods that break the linearity of traditional research methods.

Arts-based knowledge translation (ABKT) has emerged as a source of creative strategies that appropriately convey the multifaceted and interconnected nature of qualitative research in socially-grounded areas such as dementia. ${ }^{[18]}$ The use of art provides researchers with the opportunity to successfully convey complex research-based ideas to nontechnical audiences, who are better able to appreciate the design compared to academic writing. ${ }^{[19]}$ ABKT approaches in the healthcare field can be used to promote understanding of the subjective aspects of illnesses, including dementia, and evoke emotional responses among viewers by providing an embodying experience. ${ }^{[20]}$

Previous studies focused on a single artform, such as visual arts, dance and choreography, and $360^{\circ}$ videos and virtual reality. ${ }^{[18]}$ To our knowledge, no such study explored the use of multiple artforms to determine the effectiveness of one artform over the other. New media technology such as augmented reality and $360^{\circ}$ videos, for example, offer the ability to construct stories that are immersive for audiences and thereby "transform passive observers into active participants". ${ }^{[21]}$ Literature surrounding an arts-informed approach in the field of health supports the use of media as a tool for health literacy. ${ }^{[18]}$

ABKT is circular, as opposed to the linear nature of traditional translation strategies, and promotes dialogue and critical reflexivity among viewers, especially in health care providers. ${ }^{[22]}$ Parson and Boydell (2012) ${ }^{[20]}$ mentions of an audio-visual installation that helped marginalized populations become visible to healthcare providers and evoked empathy. Studies in new media for ABKT strategies have shown that media can enhance one's sense of presence, which produces a calming effect and reduces disruptive behaviour. ${ }^{[23]}$ These studies show the potential for incorporating new media technologies in furthering research and interventions for persons living with dementia. All in all, the use of ABKT can lead to direct changes in healthcare provision or indirect changes to practice facilitated by enlightened perspectives that better understand the experiences of patients. ${ }^{[20]}$ Rieger and Schultz (2014) ${ }^{[22]}$ for example, mentions that ABKT explores the meaning of illness for the patients and allows for the conveyance of emotions, creating a higher level of awareness in audiences, and engaging them in humanistic ways to better understand those being studied. ${ }^{[20]}$ Ultimately, ABKT involves adding a significant and highly needed emotional component to scholarly work that can help to challenge prevalent misconceptions regarding dementia. ${ }^{[2]}$ The use of art-based exhibits are key to promoting awareness of dementia and other socially relevant topics among viewers, and challenge their perspectives to produce clearer holistic visions of research topics. ${ }^{[24]}$

\section{METHOD}

\subsection{Research design}

A mixed-methods approach was taken to understand youth and intergenerational perspectives, attitudes, and knowledge regarding dementia and the effectiveness of art in dementia knowledge translation. A sequential explanatory design was used to collect and analyse quantitative data followed by a collection of data and its analysis of qualitative data. The qualitative results were used to assist in the explanation and interpretation of the findings of the quantitative data. ${ }^{[25]}$

\subsection{Modalities of art}

The conceptualization and creation of the art pieces were based on the themes identified in Phase 1 and 2 of the study. Phase 1 used cameras to predict agitation using multimodal sensors by track such as, heart rate, restlessness and motion. During Phase 2, a group of clinician researchers were asked to analyze videos from Phase 1 and recorded their findings using the Dementia Observation System (DOS), a tool developed specifically to capture key aspects of agitation episodes. Interrater reliability was then established between researcher findings and predictive system results for the detection of agitation.

Coded data from Phases 1 and 2 was then categorized and used to construct art pieces centered on fictional narratives to display in an art gallery. The production team consisted of a group of students from Ryerson University. The producers worked closely with the research team to generate ideas for development. The final products consisted of photographs, 
paintings, abstract data visualizations, virtual reality video and augmented reality. These art and media displays showed no confidential or identifying information of the participants from the study. The pieces were displayed at the RBC iDAPT Innovation Gallery at the Toronto Rehabilitation Institute from October to November of 2018.
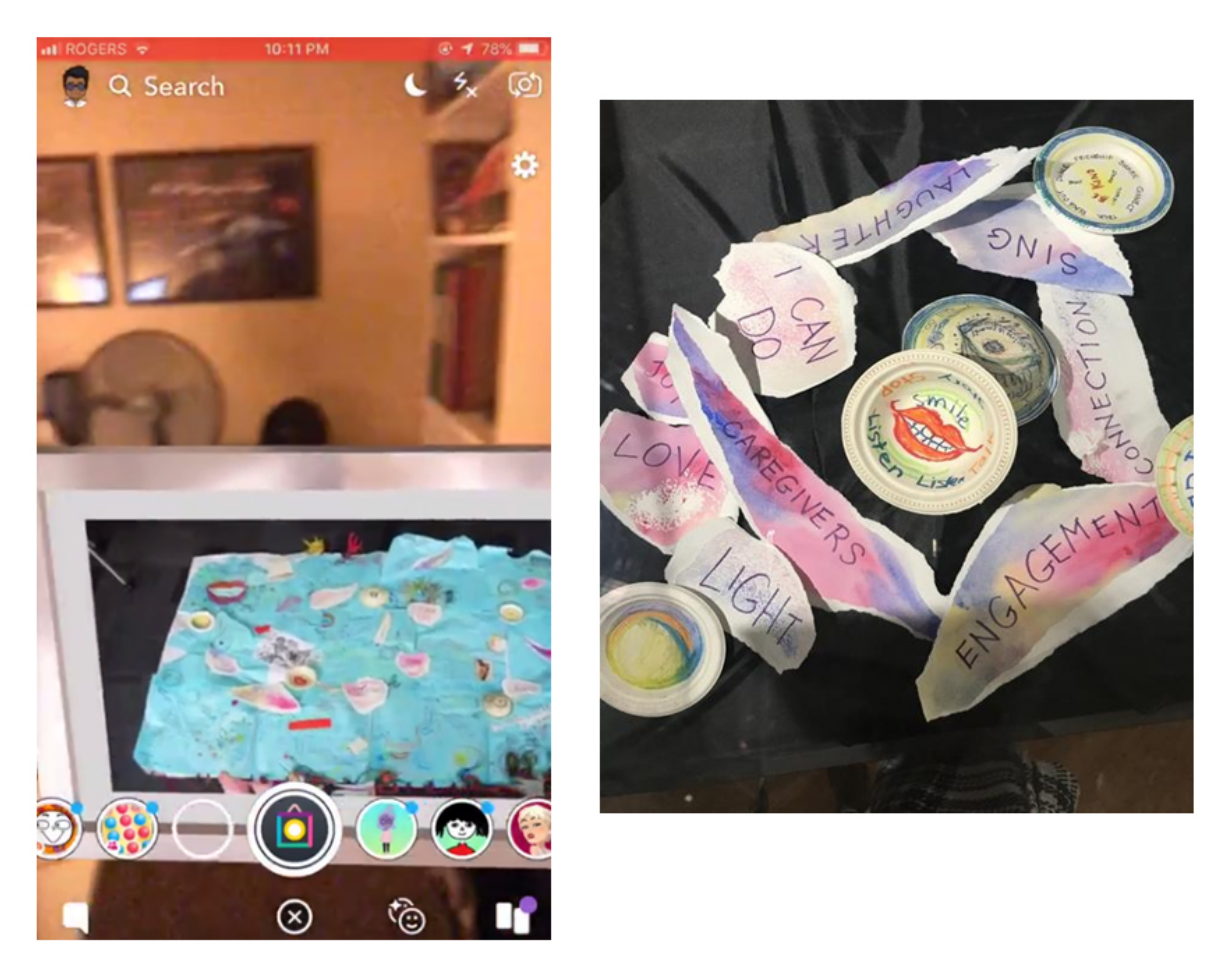

\subsubsection{Augmented Reality}

Layers of digital content and media were augmented atop physical artifacts gathered from previous studies. These objects were messages on paper and crafts created from a young carer's symposia. The overlaying content were video recordings from the symposium. Viewers were able to access this digital content by using SnapChat to scan the items - either with their own phones, or an iPad that was provided at the exhibit.

Figure 1. Please add the figure title Description: Screenshot showing the virtual gallery seen when using the Snapchat feature (created by Keenan Mathura). On the right are the physical artefacts that were the "trigger" for the virtual gallery.

\subsection{2 $360^{\circ}$ Videos}

Based on the videos collected in Phase 1, a team of creative students drafted a storyboard that infused the themes (provided by the research team). The team then worked with professional actors to record two short vignettes using a 360 camera. Both storylines were similar, but the actors were instructed to recreate certain agitated behaviours based on sexes. Charles, our male character, showed more physical agitation and fluctuations in his voice. Nina, our female character, was timid and afraid. The final videos were edited to infuse effects of darkness, blurriness, and other visual distortions to mimic (albeit not $100 \%$ accurate) certain episodes of BPSD. These videos were then rendered and imported into the Oculus Go virtual reality goggles that were a part of the exhibit. These videos can be viewed in the following link:
1) Nina:https://drive.google.com/file/d/1sp97s jsGd_RZbvIjLY149U1r2NilMhVz/view?usp=sharing

2) Charles: https://drive.google.com/open?id=1sj d68JfXG6uV85ZvxDLcfwckf5bIyyNX

\subsubsection{Abstract Data Visualizations}

Working with the researchers, another student interpreted wristband and sensor data collected from the patients and plotted them against a time-heart rate plot graph. The visualizations again were not meant to be statistically accurate, but rather abstract representations of the themes observed from early-stage data analyses. In total, we had five visualizations that represented patients' 1) increased heart rate; 2) volume fluctuations; 3) erratic movement speed; 4) need/search for order, and; 5) fidgeting. 


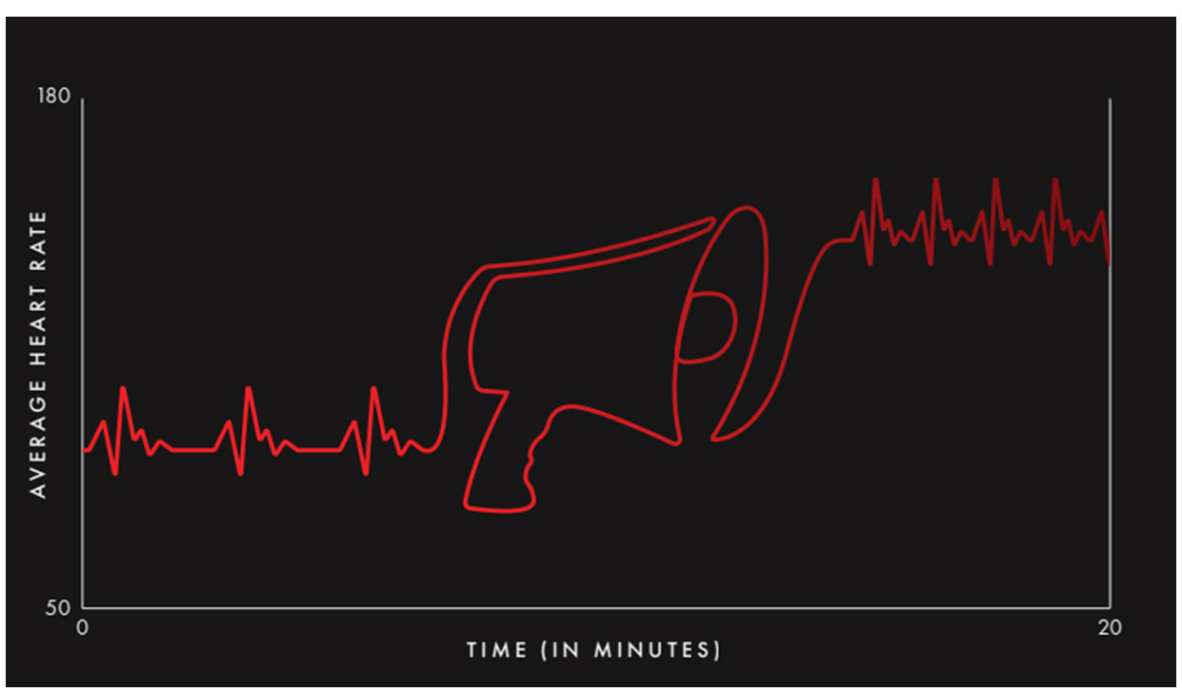

Figure 2. Please add the figure title Description: These visualizations highlight key findings from sensory data in an abstract format that is interpretable by all readers (created by Keenan Mathura).

\subsubsection{Photographs}

Using actors from the $360^{\circ}$ videos, a student photographer staged a photoshoot and instructed the actors to embody BSPD. In total, the student took over 100 photographs between both Charles and Nina and applied the doubleexposure photography technique to edit and produce the final photos. This effectively juxtaposed multiple photos into one, creating an illusion of "the seen and unseen" emotional and psychological states of a person living with dementia.

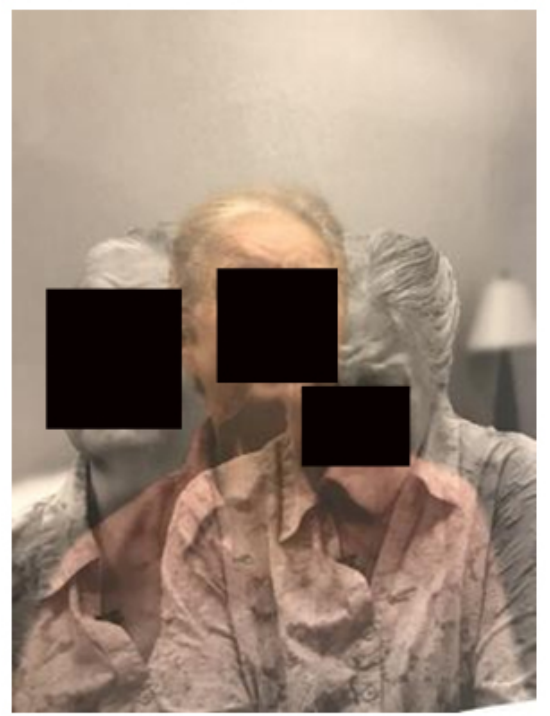

To amplify this effect, the student applied various levels of transparency and opacity of the "unseen" states, grayed out the unseen portraits, and colourized a single frame to emulate "the seen." Each of the final photos were then framed into a photo gallery. It is important to note that, across all media, the creative effects, direction, and choices were entirely the student producers. Ideas were pitched to the research team for consultation, but the production team had creative freedom to conceptualize their pieces.

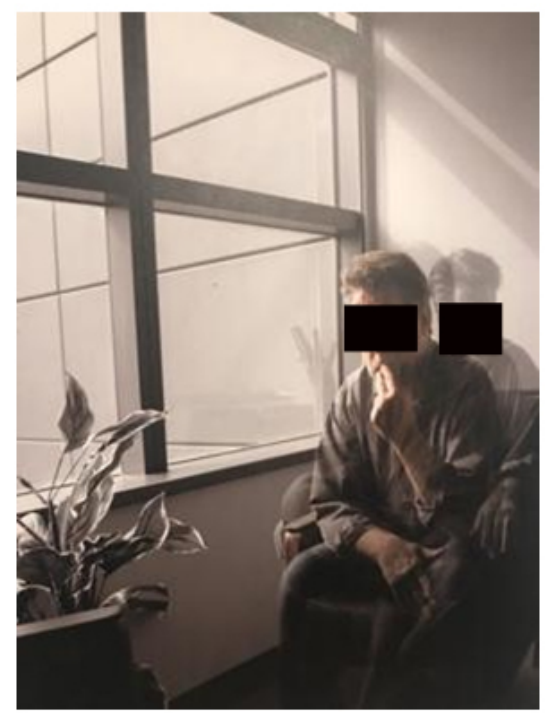

Figure 3. Photographs. Description: Multiple photos were taken on Charles and Nina to create an illusion of the seen and unseen sides of living with dementia (photography and production by Akos Katona). 


\subsubsection{Paintings}

A nursing student, who happened to be a painter, was commissioned by the research team to interpret the themes from Phase 2 of the project. The student was asked to represent, through colour and texture, themes of angst, anger, hope, confusion, violent outbursts, desperation, and other themes. Like other student producers, the student painter was given free rein on the concept development, and presented two drafts to the research team. Using mixed-media on canvas, the student used oil paint for deep and rich colour combined with other materials such as paper, styrofoam, and nuts and bolts to create various effects. By using oil paint, the student was able to incorporate feedback by painting over previous parts. In all, the student produced three main pieces, and a fourth "living piece" that invited participants to contribute their own thoughts and reflections through writing on paper and pinning their comments to the canvas.
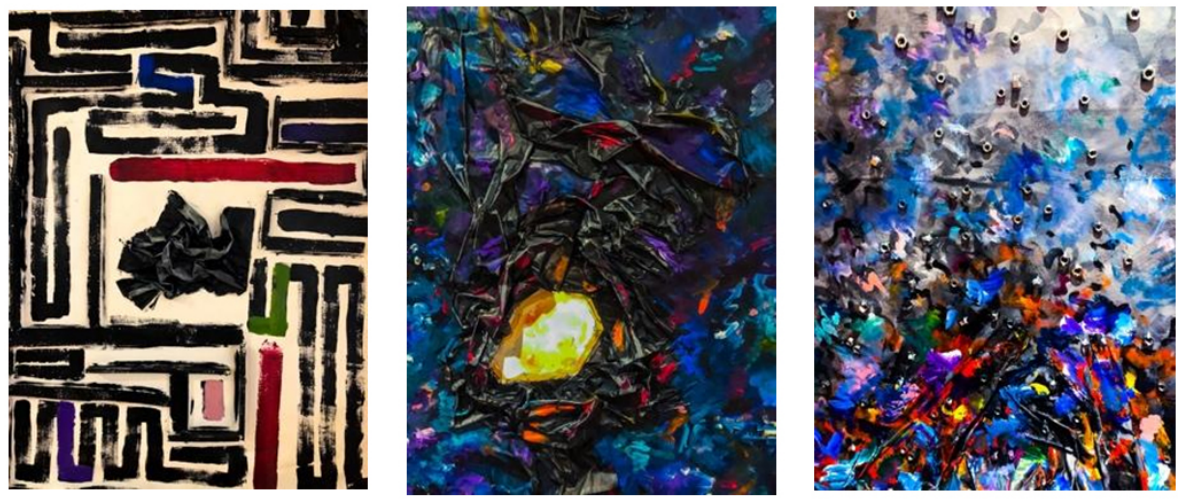

Figure 4. Paintings. Description: These three paintings used oil paint to create rich color with varying contrast. These paintings are meant to be reflexive and to be interpreted by audiences on what is it like to live with dementia (created by Hilary Tang).

\subsection{Measures}

The evaluation instruments consisted of a post-exhibit feedback survey card, Dementia Awareness Scale, participant observation and in-person interviews.

\subsubsection{Feedback survey cards}

The feedback survey card consisted of five qualitative statements with a six-point Likert Scale response option. Examples of the items on this survey card included "I enjoyed my visit to the exhibit", "I learned something new about dementia", and "the artwork enhanced my understanding of dementia". These statements were constructed with the intention to explore the level of enjoyment, depth of learning and effectiveness of technology in fostering new knowledge. All survey cards were completed anonymously by exhibit attendees who consented including patients, families, the general public and university students. Results of the survey cards were coded quantitatively. Survey cards were completely anonymous and voluntary.

\subsubsection{Observational notes}

Members of our research team randomly selected exhibit viewers to observe. Field notes were recorded using pen and paper on a chart, and were solely based on interactions; no identifying information - including sex of the viewer - were recorded. The purpose of the field notes was to record the

Published by Sciedu Press amount of time each visitor spent at each piece. Participants' body posture and interactions during the viewing of the exhibit were also documented and later categorized. Data were transcribed and coded quantitatively to examine how viewers responded to specific media.

\subsubsection{Pre/Post Dementia Awareness Scale}

O'Connor and McFadden (2010)'s Dementia Awareness Scale (DAS) is a well validated seven-point Likert scale ( $1=$ strongly disagree, $2=$ disagree, $3=$ slightly disagree, $4=$ neutral, 5=slightly agree, $6=$ agree, $7=$ strongly agree) that required respondents to rate their level of agreement with 20 qualitative statements. A higher score on the scale indicated a more positive attitude and perspective towards persons living with dementia. Examples of the statements on this self-report tool included "it is rewarding to work with people who have Alzheimer's disease-related dementias (ADRD)", "I am afraid of people with ADRD" and "I feel confident around people with ADRD". DAS forms were administered before and after viewing the exhibit. The results were coded quantitatively to evaluate any changes in self-reported attitudes towards persons living with dementia.

\subsubsection{Interviews}

Study participants were recruited to a semi-structured interview upon the completion of the post-exhibit DAS form. A 
total of 9 one-to-one interviews were conducted with nursing students. The interviews were audio-recorded and lasted about 30 to 45 minutes. Interview transcripts were analyzed qualitatively via thematic and content analyses.

\subsubsection{Demographic survey}

Demographic surveys were administered in conjunction to the interview and Dementia Awareness Scale to nursing students. All data on the demographic surveys were deidentified and labelled with a study ID number to correspond with the completed pre/post Dementia Awareness Scale forms. Components of the demographic survey consisted of age, sex, level of education and if participants knew anyone living with dementia.

\subsection{Sampling procedure}

Those who attended the exhibit from October to November of 2018 were potential subjects of the study. Physical promotional posters of the exhibit were displayed at various physical locations at Toronto Rehabilitation Institute. Specific recruitment efforts were made to students attending Ryerson University Faculty of Community Services via in class announcements and email invitations. Students were provided with a digital document outlining the study purpose and consent to participate in DAS, demographic and interview components of the study in addition to the feedback surveys that were opened to the general public. In this regard, a convenience sampling procedure was used to recruit subjects to participate in the feedback survey and purposive sampling method was used to recruit students. The participation in the study was voluntary and anonymous. Approvals from TRI and Ryerson University Research Ethics Boards were received and participant consents were obtained prior to data collection.

\subsection{Participant characteristics}

The participants of the study can be divided into university students at Ryerson University Faculty of Community Services and the general public who visited the exhibit. The inclusion criteria for participating in feedback survey card included (1) viewed the exhibit, (2) can speak, read and understand English, (3) 16 years or older.

The inclusion criteria for participation in the interview, DAS and demographic data included (1) viewed the exhibit, (2) can speak, read and understand English, (3) enrolled in the nursing or communication program at Ryerson University and (4) 18 years or older.

\section{ReSUlts}

\subsection{Recruitment analyses}

Sampling by convenience resulted in a total of 47 participants in feedback surveys, 13 Dementia Awareness Scale (DAS) surveys, and 32 structured observations. In conjunction to this pre- and post-assessment, we were able to collect 8 demographic forms. $75 \%$ of DAS participants were between the ages of 18-25 and the remaining were 25-34 years old. Due to attrition, not all participants were able to complete the post-exhibit interviews to gather their thoughts and experiences. In total, we conducted nine one-on-one interviews. All respondents of our demographic information forms were current students completing their bachelor's degree. Out of the total 13 DAS forms, 2 were excluded from data analysis due to incompletion of the items on the DAS surveys.

\subsection{Ancillary analyses}

Through the use of various data collection methods, we aimed to gather a variety of data, such as demographic, changes in dementia awareness, overall experience with ABKT, that would collectively provide a richer understanding of the impact of ABKT in raising awareness of dementia. Our study involved the use of multiple media formats to translate Phase 1 and 2 findings, making it crucial to collect unique data points that would highlight the individual impact of the art pieces. We also conducted data collection at differing points in time, with Pre- and Post-Dementia Awareness Scales and interviews post-exhibit viewing, to understand changes in participant knowledge of dementia.

\subsubsection{Demographic survey}

Collecting demographic information was important because it would help contextualize any findings of this study. For example, if this study concluded that Virtual Reality was the best way to learn, and $100 \%$ of our participants were 18-24 years old, then we can confidently confirm the findings with this age group. In total, we collected eight demographic forms, with $75 \%$ being in the 18-24 years old age range. The remaining 25\% were 25-34 years old. All respondents were current students completing their Bachelor's degree. A majority of the respondents (5/8) students knew someone living with Dementia, one of which reported that this person is a loved one or friend, and the rest are from a professional healthcare provider relationship. Seven out of eight respondents identify as female and one participant did not state. Knowing these factors helped us contextualize findings from pre/post DAS surveys, interviews, and ultimately, study results.

\subsubsection{Pre/Post Dementia Awareness Scale}

Prior to viewing the exhibit, nursing student participants were asked to complete a Dementia Awareness Scale (DAS) 
to benchmark their perceived understanding and attitudes of ADRD. The same nursing student participants were then asked to complete the same DAS after viewing the exhibit. Results from the pre/post-exhibit DAS shows that there was only a slight change in attitude when it comes to people working with ADRD. After viewing the exhibit, most visitors felt more comfortable working with people with ADRD. Two respondents reported a negative change in attitudes (i.e., they felt less comfortable). The DAS also reported a significant change in at least one participant, where upon viewing the exhibit, they felt more afraid of working with people with ADRD. All but two respondents felt more confident around people with ADRD. Although all participants reported preexhibit that they understood every person with ADRD has different needs, the post-exhibit DAS shows that this understanding was solidified and enhanced, moving from agree to strongly agree.

Findings from the pre/post-exhibit DAS were consistent in that, most participants reported a better understanding or empathy toward people with ADRD and/or working with people with ADRD after viewing the exhibit. Our data also consistently shows that a small minority of participants experienced a negative change in attitudes, specifically pertaining to the fear or discomfort of working with people with ADRD. Respondents also indicated that, prior to the exhibit, they would avoid an agitated person with ADRD. After the exhibit, most participants indicated that they would not avoid an agitated person with ADRD.

\subsubsection{Post-Exhibit Experience/Feedback Surveys}

Overall sentiment collected from the feedback forms was positive, with $87 \%$ of the participants stating that the exhibit was engaging and interactive. The use of artwork in communicating knowledge was somewhat effective: Although the majority of visitors enjoyed their visit and thought that the exhibit was engaging and interactive, a smaller percentage agreed that they had learned something new. Seventy percent of visitors agreed that the use of technology enhanced their understanding of dementia, and a higher percentage $(77 \%)$ agreed that the use of artwork, in general, enhanced their understanding of dementia.

Some participants also felt dizzy while viewing the $360^{\circ}$ videos. Although this is a known side-effect, our team forewarned participants and instructed them to stay stationary, and immediately remove the goggles if they became disoriented. Chairs were nearby in case of dizziness and the research team monitored participants for any safety hazards.

\subsubsection{Observational Notes}

Based on our observations, the most time spent were on $360^{\circ}$ videos which was on average 6 minutes, compared to other sections of the exhibit which were on average 1-2 minutes. This also created much more engagement among groups where they discussed, albeit briefly, their experiences with the $360^{\circ}$ videos. The paintings also showed some engagement, with a handful of participants discussing their experience with friends. The paintings were meant to be abstract, interpretive, and reflexive, so the team expected some deliberation among participants. Visualizations sparked the least amount of interactions: only two people spoke to a friend, and only nine people were observed as looking around. During the photography section of the exhibit, people were seen thinking and looking around; some spoke to a friend. Members of our team received positive comments about these photos, specifically that the techniques used to create the pieces were interesting.

\subsubsection{Interviews}

Our team qualitatively coded each of the nine interview transcripts to generate an overall sense of how the exhibit and its individual pieces were received. Most questions were close-ended questions that allowed us to assign numeric codes. This helped us categorize the responses and deduce general conclusions during a subsequent content analysis. We employed inductive coding that allowed us to tally repeated keywords across the nine interviews. Although these questions were not asked during the original interviews, the common keywords gave the research team a more in-depth understanding of how the exhibit was generally received. In general, interviewees shared the same positive sentiment toward the exhibit. All interviewees thought that the exhibit was effective in portraying the dementia experience, and everyone agreed that the exhibit had broadened their understanding of dementia. One respondent noted that the exhibit "...puts a perspective from the patient and not from- like a healthcare provider...and it just like expands your understanding of [dementia]." When asked whether they thought the exhibit or its individual components could be incorporated into their curricula, 7/9 said yes. In one instance, a group of students had a discussion in class of their visit to the exhibit. According to one of our interviewees, this "caused discussion of...just using artforms as a way to embody patients' experience." In reflecting on how the exhibit impacted their experience, one interviewee noted that the exhibit encouraged them to be more mindful: “...just being more cautious of how I treat my patients and giving them the choice - and making sure that, even with their condition, that they don't feel powerless."

Focusing on the individual components of the exhibit, one interviewee made specific mention of the photographs' ability to convey a particular message: "I think the largest part of the exhibit that resonated with me was the pictures that showed 
the patients who were just sitting like anyone else would. But then there was like the shadow - where it would kind of show an agitated mood or like a feeling of nervousness or some sort of anxiety or distress. It definitely showed me - told me that like, the way that they were presenting isn't exactly the way that they may feel."

This was particularly interesting as it showed congruence between the artist's intention and the viewer's experience. Our team made a conscious decision to not provide descriptors under each of the exhibit pieces. Instead, we wanted to give viewers the openness and flexibility to view and interpret each piece, making this a reflexive experience based on each individual.

\section{Discussion}

Our exhibit raised awareness and understanding of BPSD and sex differences among persons with dementia. It also promoted a dialogue about dementia with nursing students and the public. Overall, we found that the arts-based knowledge translation (ABKT) approach is an effective way to communicate research information in an interactive manner with nursing students and the public. The results from our interviews, feedback forms, and pre/post-exhibit DAS surveys, show there is strong indications that ABKT techniques, particularly in new media like VR/AR, but also in traditional artforms like paintings and photography, are effective ways to engage public audiences and promote the understanding of BPSD. Some of the outcomes of the exhibit include the acknowledgement of empathy that some viewers experienced, and the level/amount of self-reflection on everyone's own personal experiences and understanding of persons with dementia.

It is important to acknowledge that although our case study showed positive results, our sample size and participation rate was lower than expected. Despite this, results from this experience is encouraging for future case studies on ABKT strategies. We attribute the low nursing student participation rate to a number of factors, including its voluntary nature, timing of the survey (which coincided with fall reading week, midterms, and field placements), and the location of the exhibit being off-campus.

Our exhibit used five different tactics for communicating knowledge: data visualization, augmented reality, 360degree videos, painting, and photography. Results from our observational notes and interviews do not verify whether one technique is more effective than another. Looking at low-tech, for example, our data visualizations were the least effective in terms of engagement, but paintings and photographs both had high levels of engagement, discussions, and mentions. On the other hand, our $360^{\circ}$ videos were a big hit, but not our augmented reality gallery. It should be noted that the level of participant engagement depends on what each medium can afford, and what each piece of media is asking viewers to do. For example, we could have built a more interactive augmented reality gallery that required viewers to actually do something rather than just look. We could have also enhanced the complexity of the data visualizations to create a more aesthetic appeal (ours were mainly single colour and simple lines on a black background).

We also found that the level of abstraction played a big role in participant engagement. The paintings and photographs were our more artistic pieces that required a lot more reflection and self-interpretation. These had more mentions in our interviews, and viewers were observed to spend a lot more time at these pieces. Data visualizations and augmented reality, however, were more concrete in giving information to viewers to receive. These exhibits had the lowest amount of mentions and the lowest-observed time spent by viewers. In the future, we should entertain the inclusion of two additional stations: a video station that played a collection of videos and presentations from the project, and a music / listening station. This may provide more interaction to the nursing student and public participants. Further, we could better incentivize and remove barriers to participation, including a smaller version at school, or incorporating a field trip, for example, with nursing students. For example, over 400 Ryerson nursing students were invited to attend the exhibit through their fourth year course but only a few came. Future projects can use more feedback and participation from nursing students to determine the efficacy of arts-based learning methods.

Moreover, we did not collect demographic information from non-students, nor did we collect DAS surveys from nonstudent participants. We expected a majority of the participants to be 18-24 years old, but also expected a few mature nursing students. Next time, we should try to correlate demographic forms to individual responses in interviews and pre/post DAS. This would have provided us with richer data to understand participant feedback.

It should be noted that we received CTV local and national coverage of the exhibit that brought forth public interest in dementia (https://www.ctvnews.ca/health/what-does-dementiafeel-like-exhibit-lets-you-live-a-day-in-the-life-1.4144058). There were several non-students who viewed the exhibit but did not participate in the study. Again, this supports the need to use arts-based knowledge translation to communicate research information in an understandable format that is engaging and interactive with the general public and nursing students. 


\section{ACKNOWLEDGEMENTS}

We would like to thank Toronto Rehabilitation Institute for hosting the exhibit and the participants who gave their time to be involved in the study. Additionally, we would like to thank volunteers who helped out during the exhibit: Cynthia $\mathrm{Ng}$, Jessica Mastrocola, Grace Ko, Julie Le, Madeline Tuxworth, Wendy (Jing Yi) Jiang, Natasha Savigna, Maryam Mohamed, and Chelsea Valino. We would also like to acknowledge the gracious funding contributions from Ryerson University through the Ryerson Creative Fund, and Alzheimer's Society Research Program (ASRP). The exhibit was thoroughly curated and supported by the efforts of Nursing students Aleksis Penna and Hilary Tang, and Akos Katona, Keenan Mathura

\section{REFERENCES}

[1] Heese K. Ageing, dementia and society - an epistemological perspective. Springerplus. 2015; 4(1): 1-20. PMid:26069868 https: //doi.org/10.1186/s40064-015-0910-1

[2] Gale SA, Acar D, Daffner KR. dementia. The American Journal of Medicine. 2018; 131(10): 1161-1169. PMid:29425707 https: //doi.org/10.1016/j.amjmed.2018.01.022

[3] Salter KL, Kothari A. Knowledge 'Translation' as social learning: negotiating the uptake of research-based knowledge in practice. BMC Medical Education. 2016; 16: 1-10. PMid:26925578 https://doi.org/10.1186/s12909-016-0585-5

[4] Lapum JL, Liu L, Church K, et al. Arts-Informed Research Dissemination in the Health Sciences: An Evaluation of Peoples' Responses to "The 7,024th Patient" Art Installation. SAGE Open; 2014. https://doi.org/10.1177/2158244014524211

[5] Lapum J, Liu L, Church K, et al. Knowledge translation capacity of arts-informed dissemination: A narrative study. Art/Research International: A Transdisciplinary Journal. 2016; 1(1): 258-282. https://doi.org/10.18432/R2BC7H

[6] Khan KS, Zhu T, Ye B, et al. DAAD: A framework for detecting agitation and aggression in people living with dementia using a novel multi-model sensor network. In 2017 IEEE International Conference on Data Mining Workshops (ICDMW) (pp. 703-710). IEEE. 2017.

[7] Ye B, Khan KS, Chikhaoui B, et al. Challenges in collecting big data in a clinical environment with vulnerable population: Lessons learned from a study using a multi-modal sensors platform. Science and Engineering Ethics. 2018; 1-20. PMid:30357559 https: //doi.org/10.1007/s11948-018-0072-y

[8] Alzheimer's Research UK. A Walk Through Dementia Virtual Reality Android App: ARUK [Internet]. Alzheimer's Research UK. 2018 [cited 2019Aug22]. Available from: https://www . alzheimersre searchuk.org/a-walk-through-dementia-news/

[9] van der Linde RM, Dening T, Matthews FE, et al. Grouping of behavioural and psychological symptoms of dementia. International Journal of Geriatric Psychiatry. 2014; 29(6): 562-568. PMid:24677112 https://doi .org/10.1002/gps . 4037

[10] van der Linde RM, Dening T, Stephan BC, et al. Longitudinal course of behavioural and psychological symptoms of dementia: systematic review. The British Journal of Psychiatry: the Jour- and Natalie Ramtahal from Ryerson University's Centre for Communicating Knowledge at FCAD. We would like to thank the Research Ethics Board at University Health Network and Ryerson University. We would also like to thank Dr. Jennifer Lapum for her innovative work and guidance in arts-based knowledge translation, and Dr. Lori Schindel Martin for her clinical expertise on BPSD, and Phase 1 and 2 research team members for their contributions for those phases of the study. We would like to thank NSE407 Daphne Cockwell School of Nursing instructors and nursing students for their involvement.

\section{CONFLICTS OF INTEREST Disclosure}

The authors declare that there is no conflict of interest. nal of Mental Science. 2016; 209(5): 366-377. PMid:27491532 https://doi.org/10.1192/bjp.bp.114.148403

[11] Gobinath AR, Choleris E, Galea LAM. Sex, hormones, and genotype interact to influence psychiatric disease, treatment, and behavioral research. Journal of Neuroscience research. 2017; 95: 50-64. PMid:27870452 https://doi.org/10.1002/jnr. 23872

[12] Kitamura T, Kitamura M, Hino S, et al. Gender differences in clinical manifestations and outcomes among hospitalized patients with behavioral and psychological symptoms of dementia. J Clin Psychiatry. 2012; 73(12): 1548-1554. PMid:23290328 https://doi.org/10 $.4088 / \mathrm{JCP} .11 \mathrm{~m} 07614$

[13] Abi-Jaoude E, Stall NM, Rochon PA. Psychotropic drugs for the behavioural and psychological symptoms of dementia: no free ride. Canadian Medical Association Journal. 2018; 190(47). PMid:30478214 https ://doi .org/10.1503/cmaj . 181486

[14] Cousins JM, Bereznicki LR, Cooling NB, et al. Prescribing of psychotropic medication for nursing home residents with dementia: a general practitioner survey. Clinical Interventions in Aging. 2017; 12: 1573-1578. PMid:29042758 https://doi .org/10.2147/CI A. $\mathrm{S} 146613$

[15] Jennings AA, Foley T, Walsh KA, et al. General practitioners' knowledge, attitudes, and experiences of managing behavioural and psychological symptoms of dementia: A mixed-methods systematic review. International Journal of Geriatric Psychiatry. 2018; 33(9): 1163-1176. PMid:29900592 https://doi .org/10.1002/gps . 4918

[16] Bickford B, Daley S, Sleater G, et al. Understanding compassion for people with dementia in medical and nursing students. BMC Medical Education. 2019; 19(1):35-8. PMid:30683079 https ://doi .org/ 10.1186/s12909-019-1460-y

[17] Zeilig H, Poland F, Fox C, et al. The arts in dementia care education: a developmental study. Journal of Public Mental Health. 2015; 14(1): 18-23. https://doi.org/10.1108/JPMH-06-2014-0028

[18] Boydell KM, Gladstone BM, Volpe T, et al. The Production and Dissemination of Knowledge: A Scoping Review of Arts-Based Health Research [40 paragraphs]. Forum Qualitative Sozialforschung/Forum: Qualitative Social Research. 2012; 13(1): Art. 32.

[19] Simeone L, Secundo G, Schiuma G. Knowledge translation mechanisms in open innovation: The role of design in R\&D projects. Journal of Knowledge Management. 2017; 21(6): 1406-1429. https : //doi.org/10.1108/JKM-10-2016-0432 
[20] Parsons K, Boydell K. Arts-based research and knowledge translation: some key concerns for health-care professionals. Journal of Professional Care. 2012; 26(3): 170-172. PMid:22458425 https : //doi.org/10.3109/13561820.2011.647128

[21] Sylaiou S, Mania K, Karoulis A, et al. Exploring the relationship between presence and enjoyment in a virtual museum, International Journal of Human-Computer Studies. 2010; 68(5): 243-253. https://doi.org/10.1016/j.ijhcs.2009.11.002

[22] Rieger K, Schultz AS. Exploring Arts-Based Knowledge Translation: Sharing Research Findings Through Performing the Patterns, Rehearsing the Results, Staging the Synthesis. Worldviews on Evidence-Based Nursing. 2014; 11(2): 133-139. PMid:24597543 https://doi.org/10.1111/wvn.12031
[23] Garcia L, Kartolo A, Methot-Curtis E. A Discussion of the Use of Virtual Reality in Dementia, Virtual Reality in Psychological, Medical and Pedagogical Applications, Dr. Christiane Eichenberg (Ed.), InTech. 2012. Available from: http://www.intechopen .com/books/virtual-reality-in-psychological-medic al-and-pedagogical-applications/a-discussion-of-t he-use-of-virtual-reality-in-dementia

[24] Bartlett R. Visualising dementia activism: using the arts to communicate research findings. Qualitative Research. 2013; 15(6): 755-768. https://doi.org/10.1177/1468794114567493

[25] Creswell JW. Research design: Qualitative, quantitative, and mixed method approaches. 2nd ed. Sage Publications; 2003. 